\title{
Effet de la sécheresse sur la digestibilité in vitro, la teneur en ADF et la teneur en azote de la luzerne (Medicago sativa L.)
}

\author{
G. Lemaire ${ }^{1}$, J.-L. Durand ${ }^{1}$ et M. Lila ${ }^{2}$ \\ 1 INRA, station d'écophysiologie des plantes fourragères, 86600 Lusignan; \\ 2 INRA, station d'amélioration des plantes fourragères, 86600 Lusignan, France
}

(reçu le 21 novembre 1988; accepté le 20 juillet 1989)

Résumé - Les résultats d'une étude de la qualité de fourrage de luzerne, produit en conditions sèches ou irriguées, montrent que la sécheresse provoque une augmentation de la digestibilité, corrélativement à une réduction de la teneur en ADF et un accroissement de la teneur en azote dans la biomasse aérienne, car la part des feuilles est plus élevée en culture sèche. En effet, la relation entre la composition morphologique (rapport feuilles/tiges) et le niveau de production n'est pas modifiée. Cela explique en partie l'accroissement de la valeur énergétique du fourrage.

Les courbes de dilution de l'azote dans la matière sèche, comparées aux courbes de référence et aux témoins, révèlent, par contre, que la sécheresse tend à induire un déficit de nutrition azotée compensé ou non par l'accroissement de la part des feuilles, selon le niveau de limitation de la croissance.

qualité du fourrage - digestibilité - ADF - nutrition azotée - composition morphologique - déficit hydrique

Summary - A study of the change in forage quality of lucerne grown under irrigated and non-irrigated conditions. The study was carried out during the summers of 1982 and 1983 at Lusignan in the central western part of France. Growth curves were established during 2 or 3 successive summer regrowths by harvesting small plots every week. The effects of water stress on dry matter yield (DM) are presented (Fig. 1) to show the intensity of the drought at each regrowth period. At each harvest date the aerial dry matter (ADM) was divided into stem and leaf components. The leaf/stem ratio $(L / S)$ was then calculated. Nitrogen content $(N \%)$ was determined separately on the leaf and stem components and also on the total ADM. The in vitro digestibility of dry matter (IVDDM) and the acid detergent fiber content (ADF) were determined both on total aerial dry matter and on the stem component.

The change in the measured variables was expressed either in relation to time or to dry matter accumulation. This second expression allowed us to better compare irrigated and non-irrigated conditions. The LS ratio decreased more rapidly with time on irrigated than on non-irrigated lucerne. The evolution of LS in relation to $A D M$, however, was the same under the 2 conditions and a common relationship between these 2 variables is proposed : LSS $=1.4$ (ADM)-0.56 (Fig. 2). This relationship permits the LS value to be predicted directly from dry matter yield whatever the water conditions are. The higher values of LS observed during the drought at a given time were therefore entirely due to a lower ADM accumulation than when water was non limiting.

The $N$ content of $A D M$ decreased less rapidly with time under non-irrigated conditions than under irrigated conditions. But for the same quantity of accumulated dry matter, $N$ content was lower in water-stressed than in well watered lucerne (Fig. 4), indicating a lower level of nitrogen nutrition. The global effect of drought on the forage $N$ content was therefore the result of a negative effect on the $N$ nutrition processes which was more or less compensated by a positive effect due to a lower dilution of $N$. This phenomenon allows us to explain some contradictory results in the literature which relate either positive or negative effect of water stress on the $N$ content of harvested lucerne forage.

The water stress led to an increase in IVDDM and to a decrease in ADF content of the harvested forage (Fig. 7). This effect can be explained by the increase in the LS ratio and by an increase in the digestibility of the stem component. This increase in the digestibility of stems can be directly related to the decrease of stem dry matter. $A$ general relationship between the IVDDM and the dry matter yield of lucerne forage in natural conditions is proposed as an indicator for predicting forage quality.

forage quality - digestibility - ADF - nitrogen nutrition - morphological composition - water deficit 


\section{INTRODUCTION}

La qualité d'un fourrage de luzerne est caractérisée à la fois par la digestibilité de la matière organique, qui détermine la quantité d'énergie fournie sous une forme digestible, métabolisable et nette au ruminant, et par la teneur en azote de la matière sèche, qui détermine en grande partie la teneur en protéines et la valeur azotée.

Ces 2 paramètres, digestibilité et teneur en azote, sont tous deux liés à la proportion de feuilles dans la matière sèche récoltée. Cette dernière composante, de nature morphologique, peut être exprimée par le rapport entre la masse de feuille et la masse de tige : F/T (Demarquilly, 1966). Cependant, à une même valeur de $F / T$, c'est-à-dire pour une même composition morphologique, peuvent correspondre des valeurs différentes de digestibilité ou de teneur en azote, du fait de différences de composition chimique des feuilles et des tiges.

Ainsi, l'effet d'une sécheresse sur la valeur alimentaire de la luzerne peut être analysé en étudiant séparément l'effet de la sécheresse sur la composition morphologique de la plante et son effet sur sa composition chimique (Christian et al., 1970). La teneur en azote de la plante entière est égale à la moyenne entre la teneur des tiges et la teneur des feuilles, pondérées par la masse relative de chacun des compartiments $(F / T)$. De la même manière, la teneur en ADF de la plante entière, qui détermine en grande partie la digestibilité du fourrage, peut être reconstituée à partir de la teneur en ADF des feuilles et des tiges prises séparément et de leur masse relative (Albrecht et al. 1987).

L'effet de la sécheresse sur l'évolution de $F / T$ a été signalé par les travaux de Vough \& Marten (1971), Brown \& Tanner (1983), qui ont montré une diminution moins rapide de ce rapport au cours de la croissance sur les plantes soumises à un déficit hydrique. Cela se traduit naturellement par une moindre diminution des teneurs en azote de la plante entière et par une moindre augmentation de la teneur en ADF, du fait d'une proportion accrue des feuilles dans la biomasse récoltée.

Les effets directs de la sécheresse sur la composition chimique des feuilles et des tiges prises séparément ont été beaucoup plus rarement abordés. Vough et Marten (1971) puis Snaydon (1972) ont cependant mis en évidence une augmentation de la digestibilité des tiges, accompagnée d'une diminution de leur teneur en ADF, sur des plantes soumises à un déficit hydrique en conditions contrôlées. Par contre, ces auteurs ne signalent aucun effet sur la digestibilité des feuilles ni sur leur teneur en ADF.
Les effets de la sécheresse sur la teneur en azote apparaissent souvent contradictoires. Ainsi, Vough \& Marten (1971) observent soit des augmentations, soit des diminutions de la teneur en azote des plantes subissant une sécheresse. Ces résultats sont semblables à ceux de Carter et Sheaffer (1983a), qui attribuent les variations de la teneur en azote à des variations de la capacité de fixation de l'azote de l'atmosphère. Ces différents auteurs n'ont cependant pas pris en compte simultanément les variations de teneurs en azote de la plante entière dues aux variations de leur composition morphologique et aux variations intrinsèques de teneur en azote des feuilles et des tiges.

D'autre part, les travaux de Lemaire \& Salette (1984) ont montré que pour interpréter les variations de teneur en azote il était nécessaire de tenir compte des différences de croissances. Cela du fait de la dilution de l'azote dans la biomasse élaborée au cours d'une repousse, qui conduit à une diminution de la teneur en azote d'autant plus forte que la biomasse produite est importante.

Dans le but d'analyser l'effet de la sécheresse sur les différents composants de la qualité énergétique et azotée de la luzerne, nous avons mis en place une expérimentation comprenant des parcelles sèches et des parcelles irriguées, sur lesquelles nous avons suivi simultanément, au cours des différentes repousses, les dynamiques de croissance en matière sèche et d'évolution des critères de qualité : évolution du rapport $F / T$, des teneurs en azote des parties aériennes, des feuilles, des tiges, et de digestibilité in vitro de la plante entière et des tiges prises séparément.

\section{MATÉRIELS ET MÉTHODES}

L'essai a été implanté en 1981 avec la variété Europe semée au mois d'avril à la densité de $20 \mathrm{~kg} / \mathrm{ha}$. Les traitements secs et irrigués ont été appliqués en 1982 et 1983 sur des parcelles séparées. Pour chaque année d'étude, chaque repousse était étudiée sur des parcelles différentes disposées en 4 blocs aléatoires complets (Durand et al., 1989). Des prélèvements étaient effectués chaque semaine afin d'obtenir les courbes de croissance. Chaque prélèvement correspondait à $2 \mathrm{~m}$ linéaires sur $20 \mathrm{~cm}$ de large (largeur d'une ligne), récoltés à la micro-tondeuse, $6 \mathrm{~cm}$ audessus du niveau du sol. Sur un échantillon représentatif des parties aériennes récoltées (de poids frais égal à $500 \mathrm{~g}$ ), une séparation morphologique entre feuilles et tiges et, sur chaque composante, une détermination de la teneur en azote (méthode Kjeldhal) étaient réalisées ainsi que sur un échantillon de la matière sèche aérienne non triée.

La digestibilité in vitro (méthode Tilley et Terry, 1963) ainsi que les teneurs en ADF (Van Soest, 1963) ont été mesurées pour les repousses de 1983 et sur 
une repousse en 1982, pour les tiges et pour la matière sèche aérienne.

L'irrigation était effectuée de manière à maintenir l'humidité du sol proche de la capacité au champ. Deux tubes d'accès pour la sonde à neutrons étaient placés dans chaque parcelle, nous permettant de mesurer la consommation d'eau hebdomadaire pour les différents traitements (Lemaire et Roberge, 1980).

\section{RÉSULTATS}

\section{Effets de la sécheresse sur les courbes de croissance}

La Figure 1, reprise de Durand et al. (1989), montre l'effet de la sécheresse sur les niveaux de consommation en eau et sur la croissance en matière sèche pour les différentes repousses étudiées. Trois repousses ont été affectées par la sécheresse en 1982 et une en 1983. L'intensité de la sécheresse a varié considérablement d'une $G / M^{2}$
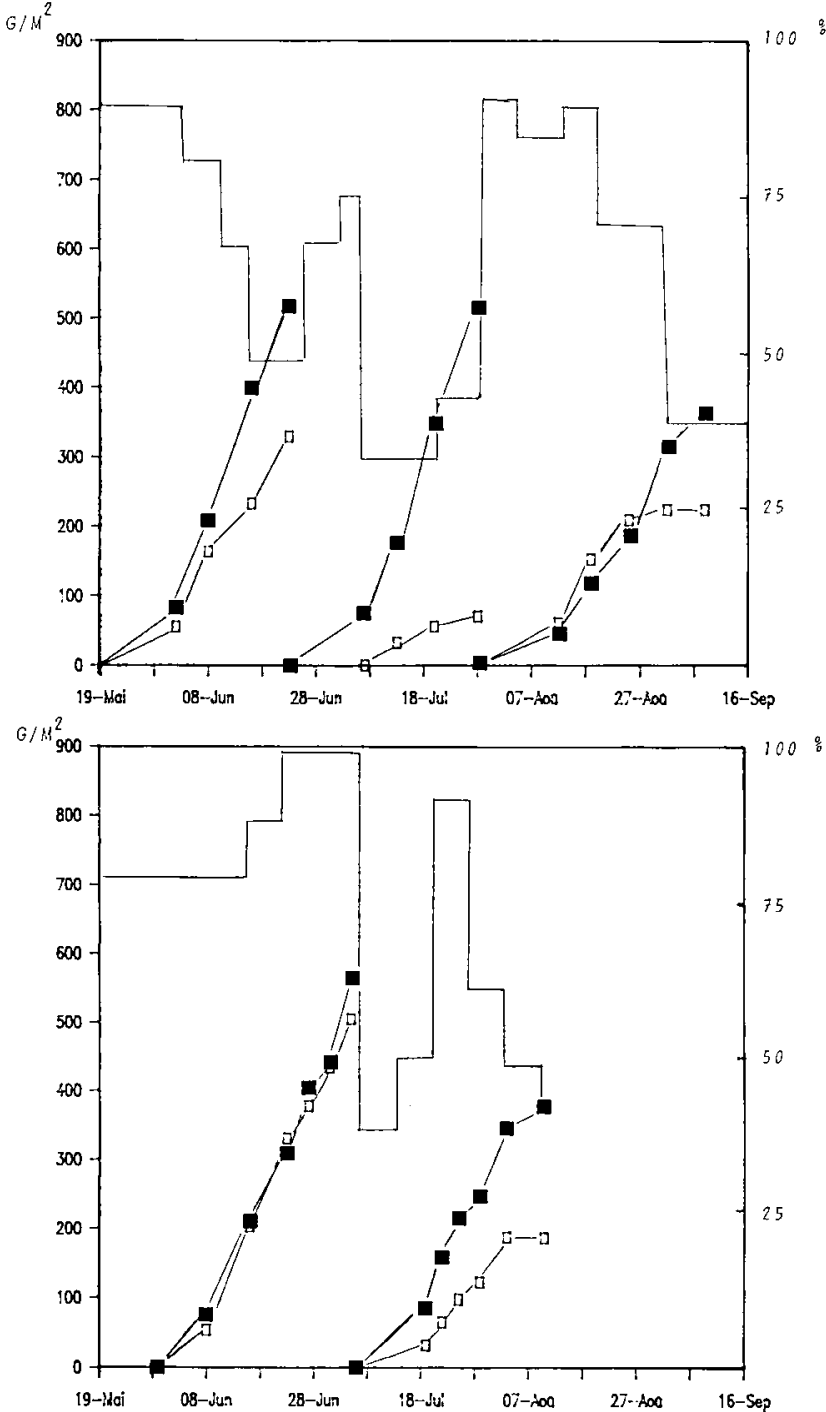

Fig. 1. Evolution de la matière sèche exportée (MSA) en 1982 (a) et 1983 (b);

$\square$ : traitement sec; $\boldsymbol{\square}$ : traitement irrigué.

$\checkmark$ : évolution du rapport entre les consommations d'eau en sec et en irrigué $\left(\mathrm{CH}_{\mathrm{sed}} / \mathrm{CH}_{\text {irrigué. }}\right)$ repousse à l'autre, atteignant parfois un taux de satisfaction des besoins en eau de moins de $50 \%$, ce qui s'est traduit par une croissance quasiment nulle au cours de la seconde repousse de 1982.

\section{Effet de la sécheresse sur la composition morphologique (rapport F/T)}

La diminution du rapport feuilles/tiges au cours de la repousse est plus faible dans les parcelles sèches que dans les parcelles irriguées, ce qui, pour la luzerne, est un résultat classique (Vough \& Marten, 1971; Snaydon, 1972; Brown \& Tanner, 1983; Carter \& Sheaffer, 1983a). II est cependant nécessaire de vérifier si cet effet de la sécheresse s'explique en totalité par la diminution de la biomasse, conformément au modèle proposé par Lemaire et al. (1985).

La Figure 2 représente l'évolution du rapport $\mathrm{F} / \mathrm{T}$ au cours des différentes repousses, exprimé en fonction de la biomasse produite. En conditions irriguées, ce rapport évolue selon une loi de la forme :

$$
\mathrm{F} / \mathrm{T}=1,4 \times(\mathrm{MSA})-0,56
$$

où MSA est la matière sèche aérienne (en t.ha-1).

Le coefficient d'allométrie de cette relation $(0,56)$ est très proche de la valeur de 0,63 déterminée par Lemaire et al. (1985). Cette relation indique que le rapport entre les vitesses relatives

$$
\left(\frac{1}{\mathrm{~F}} \times \frac{\mathrm{dF}}{\mathrm{dt}}\right) /\left(\frac{1}{\mathrm{~T}} \times \frac{\mathrm{dT}}{\mathrm{dt}}\right)
$$

de croissance des feuilles et des tiges,

reste constant au cours d'une repousse et identique d'une repousse à l'autre. Ce rapport inférieur à 1 traduit le fait que la masse de feuilles augmente moins vite que la masse de tiges.

La Figure 2 montre que la sécheresse n'affecte pas cette relation d'allométrie, qui semble

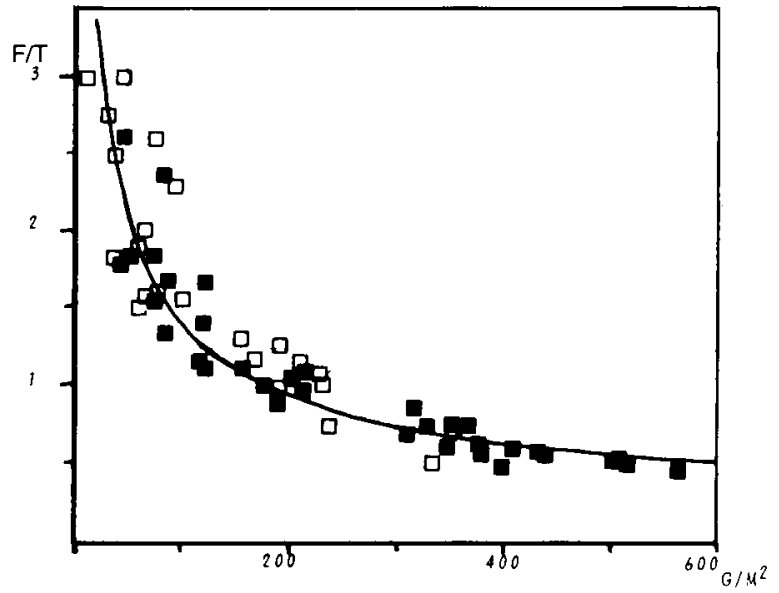

Fig. 2. Relation entre la biomasse aérienne accumulée et le rapport F/T. $\mathbf{\square}$ : irrigué; $\square$ : sec; — : ajustement linéaire; $F / T=1,40 * M S A-0,56 R^{2}=0,979$. 
avoir une valeur très générale en couvert dense (Brown \& Tanner, 1983). Ainsi, l'augmentation du rapport $F / T$ en situation sèche résulte exclusivement du ralentissement de la croissance.

\section{Effet du déficit hydrique sur la teneur en azote}

L'évolution de la teneur en azote pour les différents traitements et pour les différentes repousses est représentée sur la Figure 3. On constate que, en règle générale, l'effet de la sécheresse se traduit par une moindre diminution des teneurs en azote au cours de la croissance. A une même date, les parcelles ayant été affectées par la sécheresse ont des teneurs en azote plus élevées que les parcelles irriguées. Cela confirme les résultats de Cole et al. (1970), Snaydon (1972), Carter \& Sheaffer (1983a), qui ont montré que la sécheresse avait tendance dans certains cas à augmenter la teneur en azote de la luzerne alors que dans d'autres situations, ces teneurs n'étaient pas augmentées ou étaient même réduites.

$\mathrm{Si}$ l'on analyse l'évolution des teneurs en azote en fonction de la biomasse aérienne élabo-
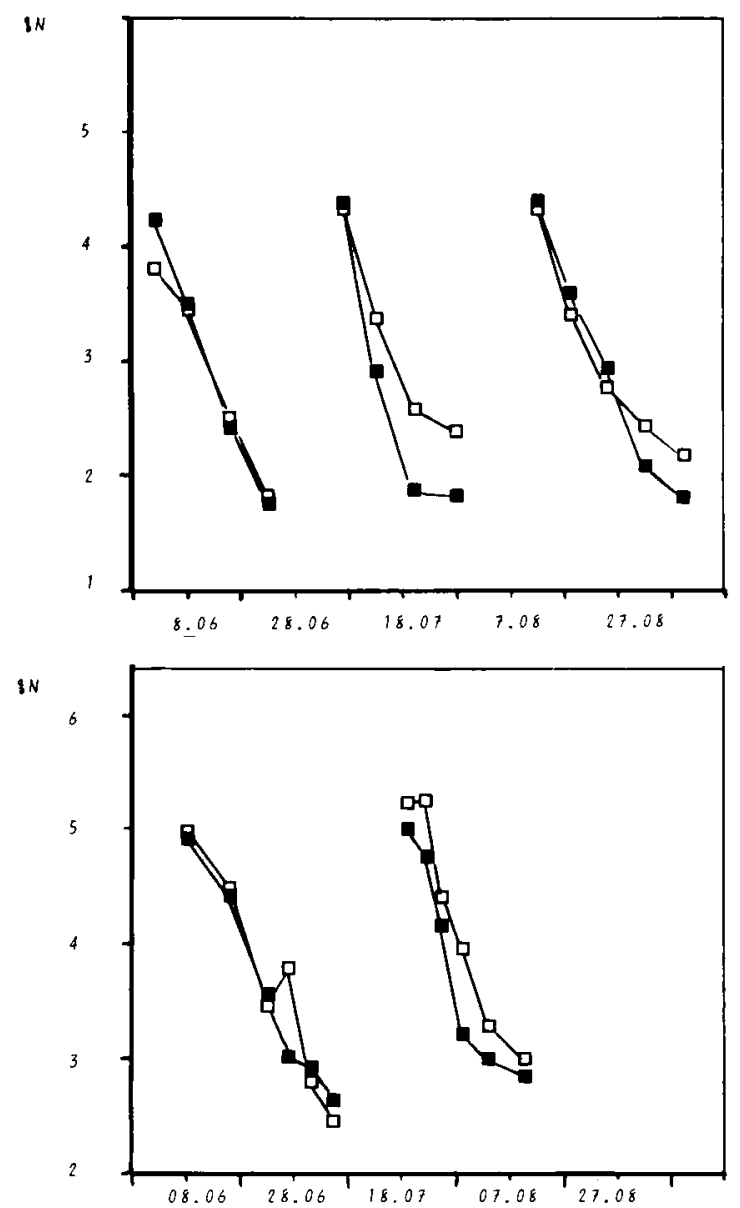

Fig. 3. Evolution de la teneur en azote de la matière sèche exportée durant les repousses de 1982 (a), et 1983 (b). rée au cours d'une repousse (Fig. $4 a$ ), on constate que, pour une même production de matière sèche aérienne, les parcelles ayant subi un déficit hydrique ont, en général, des teneurs en azote plus faibles que les parcelles irriguées correspondantes. Nous pouvons suivre l'évolution de la sécheresse sur les courbes de dilution. C'est particulièrement net sur les Figures 4 (b à f), où les traitements secs et irrigués sont comparés, pour chacune des repousses, au modèle correspondant à la courbe de dilution à azote non limitant (Lemaire et al., 1985) :

$$
\mathrm{N} \%=5(\mathrm{MSA})-0,34
$$

où $\mathrm{N} \%$ est la teneur en azote de la matière sèche et MSA la quantité de matière sèche en tonnes par hectare.

Rappelons que cette relation n'est pas valable pour des quantités de matière sèche aérienne inférieures à $1 \mathrm{t} / \mathrm{ha}(\% \mathrm{~N}$ ne tend évidemment pas vers l'infini quand la biomasse tend vers 0 ). Ainsi que l'indiquent Salette \& Lemaire (1981), les premiers stades de croissance suivant immédiatement la coupe ne doivent pas être pris en compte dans ce modèle, qui surestime la teneur en azote dans ces stades particuliers.

Les courbes établies pour les parcelles irriguées sont en règle générale plus ou moins bien superposables à la courbe de référence, tandis que les parcelles sèches présentent des teneurs en azote inférieures à la teneur optimale correspondante. Selon Lemaire \& Denoix (1987), et par analogie aux peuplements de graminées, cela doit être interprété comme la conséquence d'une limitation de la nutrition azotée induite par la sécheresse.

Cette limitation se manifeste simultanément ou postérieurement à la réduction de la vitesse de croissance des parties aériennes, selon les repousses. Cette limitation de la nutrition azotée intervient au niveau de la plante entière, alors même que le rapport $F / T$ est accru, ce qui tend à réduire les différences de teneur en azote constatées au niveau des parties aériennes.

Les données publiées par Carter \& Sheaffer (1983a) ont été reportées sur la Figure 4a. Ces auteurs exprimaient l'impossibilité de discerner un effet net de la sécheresse sur la teneur en azote, car les teneurs de tous les traitements étaient très voisines. En fait, on voit bien que le modèle de dilution de l'azote permet au contraire de mettre en évidence un déficit de nutrition azotée très important pour les parcelles cultivées sans irrigation. Ainsi, à l'effet de la sécheresse sur la croissance en matière sèche de la luzerne se superpose un effet sur sa nutrition azotée par réduction de la fixation symbiotique. La sensibilité de la fixation d'azote à la sécheresse a par 
\&N
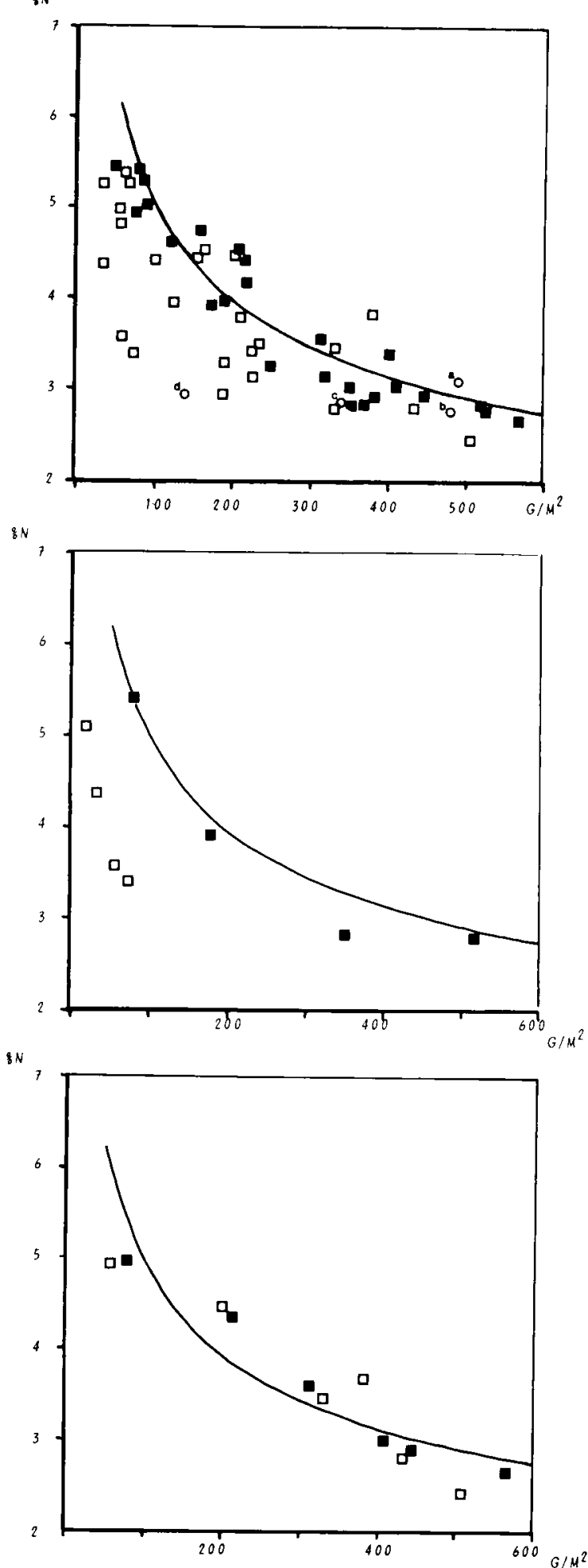



\&
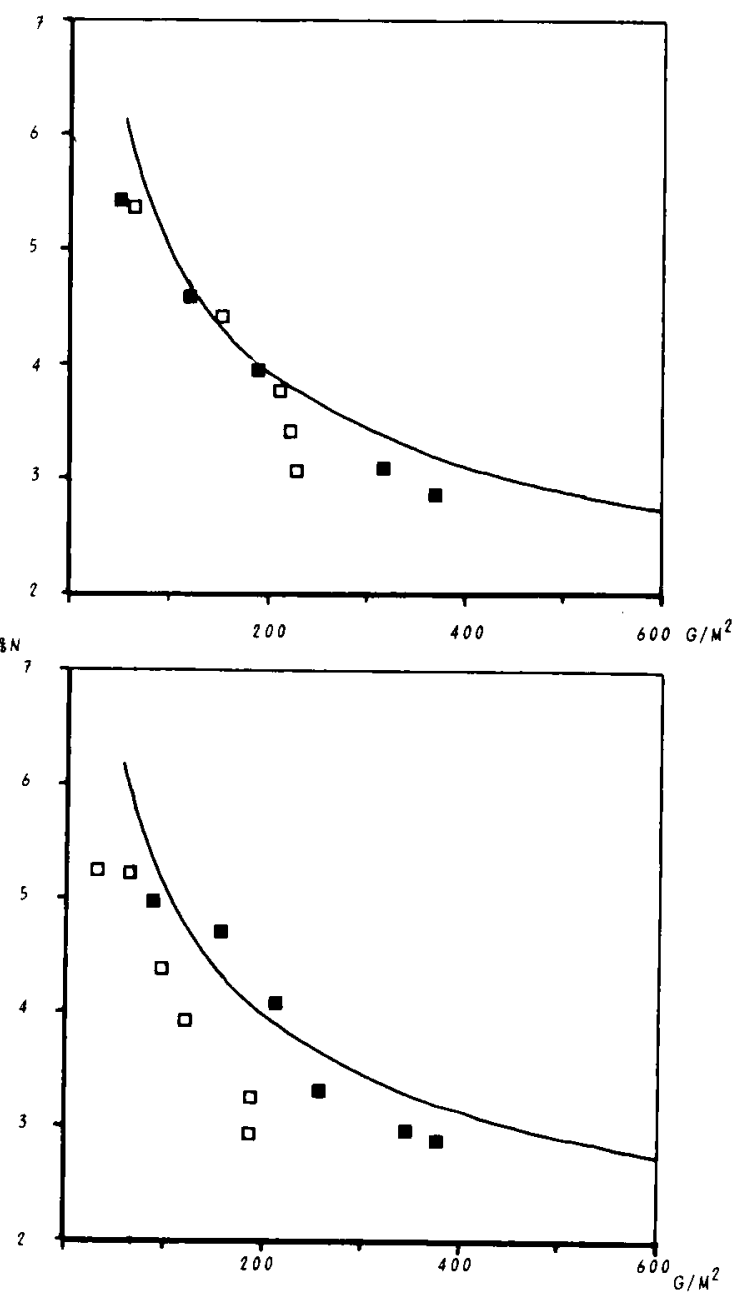

Fig. 4 a à f. Relation entre la biomasse aérienne accumulée et la teneur en azote de la matière sèche aérienne, pour l'ensemble des repousses (a); $\square$ : sec; $\square$ : irrigué; o : d'après les données des tableaux 1 et 2 in Carter et Scheaffer 1983a, traitements hydriques de plus en plus limitant de a à co. Relation par repousse (b: première repousse de 1982, c: deuxième repousse, $d$ : troisième repousse, e: première repousse de 1983 , f: deuxième repousse).

ailleurs été mesurée chez d'autres légumineuses (Sprent, 1976; Durand et al., 1987) ou chez la luzerne (Carter \& Sheaffer, 1983b; Wery, 1987).

$\mathrm{Si}$ on analyse séparément la teneur des feuilles et des tiges, on met en évidence l'effet intrinsèque de la sécheresse sur l'alimentation en azote des feuilles et des tiges. On voit sur la
Figure 5 que les teneurs en azote des feuilles et des tiges ne sont limitées que pour des niveaux d'alimentation hydrique très faibles, correspondant à une réduction de la croissance très importante.

Ainsi, au niveau de la biomasse aérienne, on peut expliquer les effets contradictoires de la 

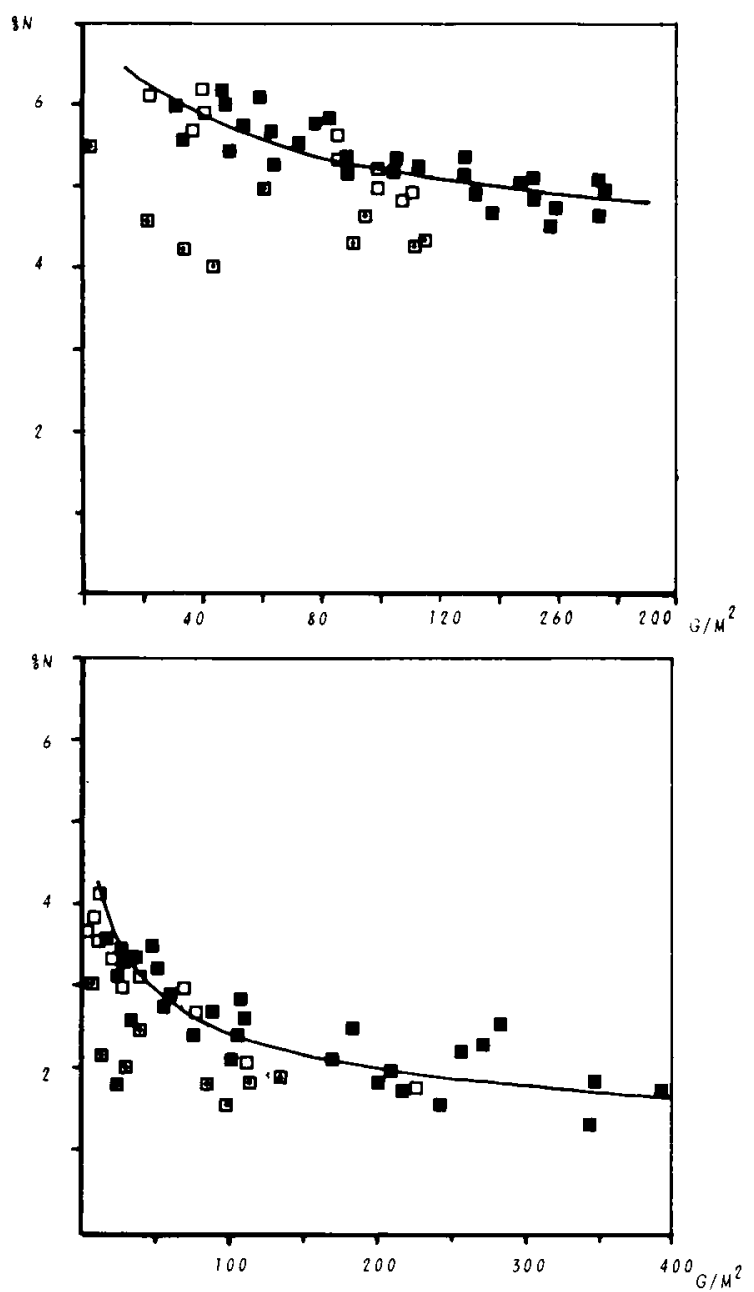

Fig. 5. Relation entre la masse de feuilles (a), et de tiges (b) et leur teneur en azote. $\square$ : points correspondant aux dates ou la MSA était différente en sec et en irriguée.

sécheresse sur la teneur en azote relevés dans la littérature : un effet négatif qui est concomitant d'une réduction de la croissance en matière sèche, et un effet positif par le biais d'un accroissement du rapport $F / T$, lui-même conséquence de la diminution de la croissance.

L'importance de la composition morphologique sur l'évolution des teneurs en azote est illustrée par la Figure 6, où celle-ci est mise en relation avec le pourcentage de feuilles dans la biomasse aérienne. En combinant les relations (1) et (2), on obtient la relation :

$$
\% \mathrm{~N}=4,08 \times\left[(\mathrm{F} / \mathrm{MSA})^{-1}-1\right]^{-0,61}
$$

où $F / M S A$ est la part des feuilles dans la matière sèche $(0<F / M S A<1)$.

Les points expérimentaux obtenus en culture irriguée se regroupent bien autour de cette relation représentée sur la Figure 6, à l'exception des points correspondant aux stades précoces (F/MSA $>0,65$ ), pour lesquels le modèle de dilution de l'azote ne s'applique pas. On observe au cours des repousses en situation sèche une diminution plus rapide des teneurs en azote que celle impliquée par la diminution progressive de la part des feuilles dans la constitution de la bio-



Fig. 6. Relation entre la part des feuilles dans la MSA et la teneur en azote de la MSA pour les parcelles non irriguées $(\diamond)$ et irriguées $(\bullet) . \quad: \% N=4,08^{*}\left[(F / M S A)^{-1}-1\right]^{-0.61}(3)$.

masse aérienne. II y a bien un effet direct de la sécheresse sur la teneur intrinsèque en azote du fourrage.

\section{Effets d'un déficit hydrique sur la valeur énergétique du fourrage}

Les teneurs en ADF du fourrage et la digestibilité in vitro sont représentés sur la Figure 7 . On constate une diminution de la teneur en ADF du fourrage ainsi qu'une augmentation de la digestibilité. II s'agit principalement d'une diminution de la digestibilité des tiges, accompagnée d'une augmentation du rapport $F / T$. On remarque que les teneurs en ADF de début de repousse sont toutefois un peu plus faibles que celles généralement indiquées (Vough \& Marten, 1971), mais sans que cela puisse être imputé à la sécheresse. Pour la digestibilité, ces résultats sont en accord avec ceux de Snaydon (1972), qui indiquait des augmentations de l'ordre de $5 \%$. Carter \& Sheaffer (1983a) cependant n'ont mesuré de tels écarts que pour des sécheresses très marquées.

La relation entre la digestibilité des tiges et la teneur en ADF a été estimée en culture sèche et en culture irriguée.

$$
\begin{array}{ll}
\mathrm{DIG}=100,4-1,06 \times \mathrm{ADF} & \mathrm{R}^{2}=0,91 \text { irrigué (3) } \\
\mathrm{DIG}=106,2-1,35 \times \mathrm{ADF} & \mathrm{R}^{2}=0,90 \mathrm{sec}
\end{array}
$$

Le test de parallélisme indique que les pentes sont différentes avec une probabilité de $10 \%$. Cela indique qu'en culture sèche, vers la fin de la repousse et à même teneur en ADF, les tiges ont tendance à être moins digestibles qu'en culture irriguée. Cette tendance, même faible, montre qu'il n'est pas possible d'utiliser une relation unique pour estimer la digestibilité à partir de l'analyse de l'ADF. Des études spécifiques seraient nécessaires pour établir comment le déficit hydrique peut modifier cette relation (arte- 

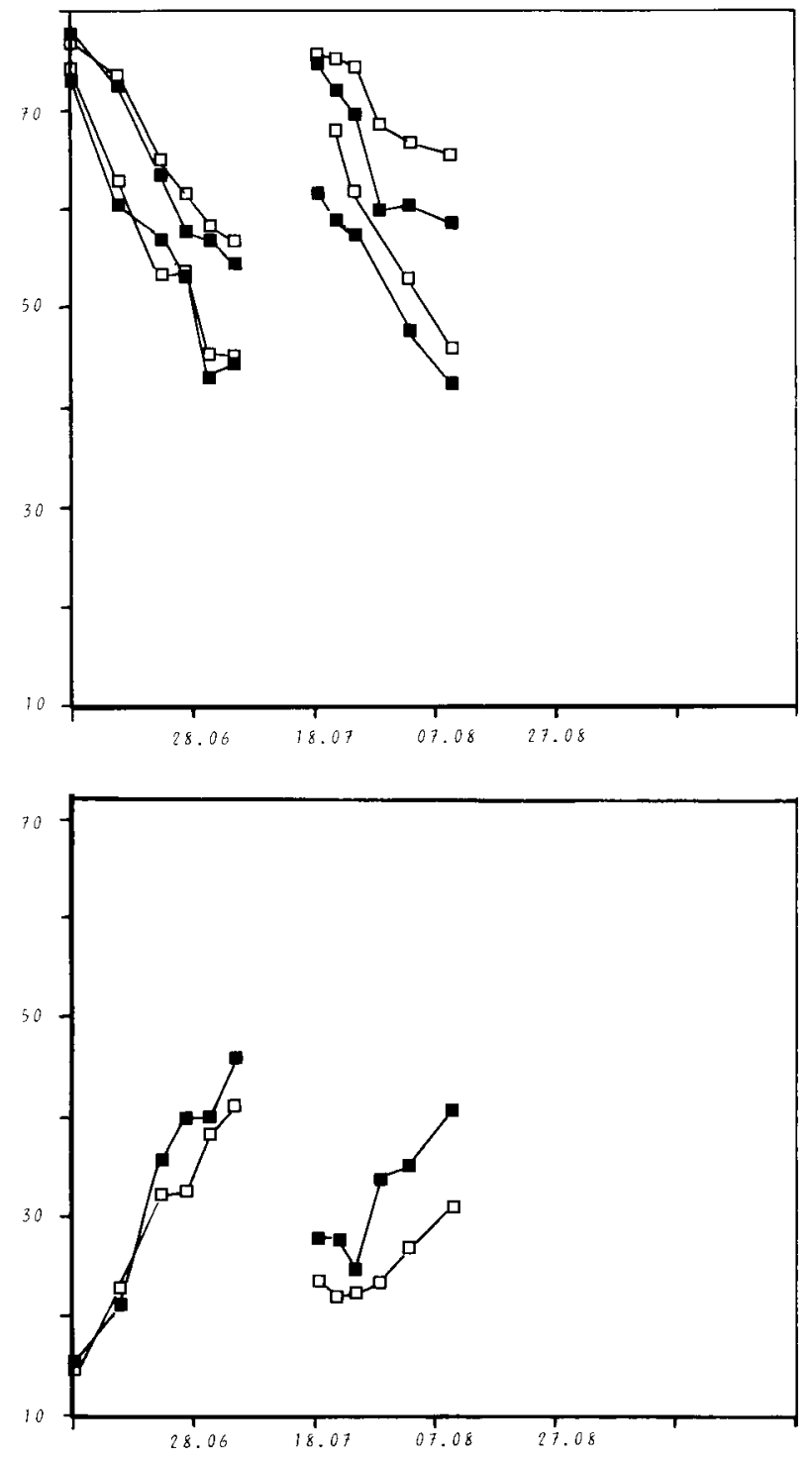

Fig. 7. Evolution de la digestibilité in vitro (a: à une même date, les valeurs inférieures concernent les tiges et les valeurs supérieures concernent le MSA) et de la teneur en ADF (b) de la MSA pour les repousses de 1983. $\square$ : sec; - : irrigué.

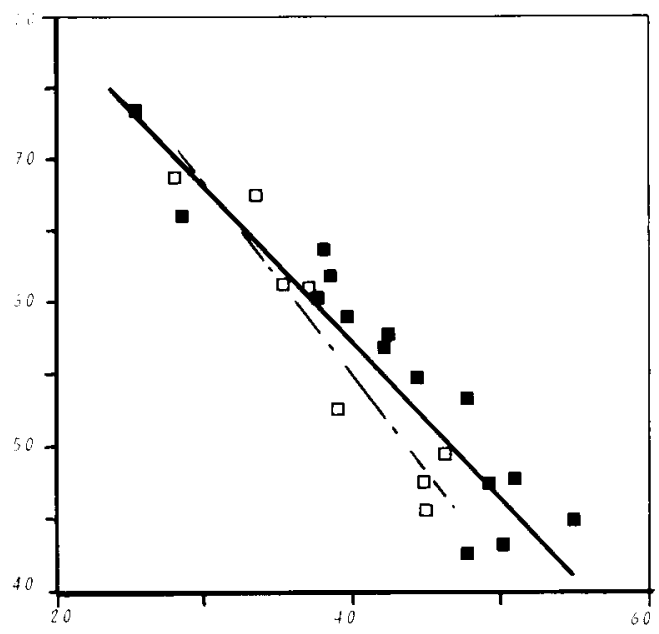

Fig. 8. Relation entre la teneur en ADF des tiges et la digestibilité. $\square$ : sec; $\mathbf{\square}$ : irrigué. fact lié au broyage, composition chimique de la fraction d'ADF, propriétés mécaniques des parois).

La relation entre la digestibilité (ou la teneur en ADF) de la matière sèche (feuilles et tiges) et le pourcentage de feuilles dans la biomasse aérienne indique, que la digestibilité, et par là même la valeur énergétique du fourrage, dépend directement de sa composition morphologique (Fig. 9). La correspondance entre ces résultats et ceux de Vough \& Marten (1971), qui donnent simultanément le pourcentage de feuille et la digestibilité, est d'ailleurs remarquable. Cette dépendance n'apparaît pas davantage modifiée par la sécheresse qu'elle ne l'était dans l'étude de ces auteurs. Cela nous permet de proposer une relation unique entre la proportion de feuilles dans la biomasse aérienne et la digestibilité de cette biomasse :

$$
D I G=65,2 \times \% F+36,5 \quad R^{2}=0,857
$$

En utilisant le rapport $F / T$, cette équation devient :

$$
\mathrm{DIG}=\left[65,2 \frac{\mathrm{F}}{\mathrm{T}} /\left(1+\frac{\mathrm{F}}{\mathrm{T}}\right)\right]+36,5
$$

En conséquence, en combinant (1) et (6), on obtient la relation univoque entre la digestibilité et la biomasse aérienne :

$$
D I G=65,2\left(\frac{1,4 \mathrm{MSA}^{-0,56}}{1+1,4 \mathrm{MSA}^{-0,56}}\right)+36,5
$$

où MSA est en tonnes par hectare et DIG en pour cent.

Cette équation pourrait permettre une estimation directe de la digestibilité de la luzerne à partir des mesures de production de matière sèche, quel que soit le niveau d'alimentation hydrique. II faudrait cependant valider une telle relation à l'ai-

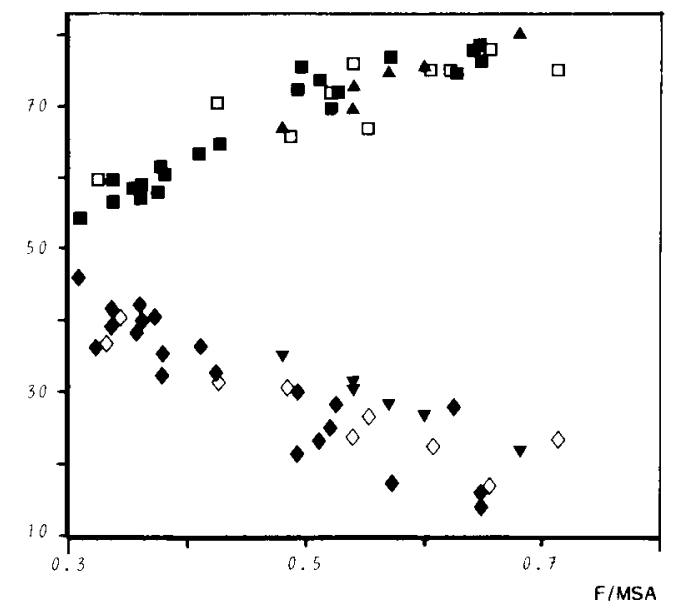

Fig. 9. Teneur en ADF ( $S$ ), et digestibilité $(\square)$ de la MSA en fonction de la part des feuilles dans la matière sèche exportée. $\boldsymbol{\nabla}$ : sec; $\boldsymbol{\nabla}$ : irrigué, $\boldsymbol{\nabla}$ et $\boldsymbol{\Delta}$ : respectivement teneur en ADF et digestibilité d'après les données du tableau 3 in Vough et Marten 1971. 
de de données complémentaires et indépendantes.

\section{CONCLUSION}

Cette analyse confirme, y compris en conditions hydriques limitantes, le rôle prépondérant que joue la composition morphologique de la luzerne dans la détermination de la qualité.

Pour une même biomasse produite, le rapport $\mathrm{F} / \mathrm{T}$ est le même en culture sèche et en culture irriguée. La digestibilité des tiges est liée négativement à la biomasse de tiges. II s'en suit une très forte corrélation positive entre la digestibilité des tiges et le rapport $F / T$. Ainsi, ce rapport détermine directement la digestibilité de la biomasse : d'une manière directe du fait de la plus grande proportion de feuilles et d'une manière corrélative par le fait que les rapports $F / T$ élevés correspondent à des entrenœuds courts et peu lignifiés. II est remarquable alors de constater qu'une même relation puisse rendre compte à la fois de l'évolution de la digestibilité due à l'âge et de celle due à la croissance. Cela pose le problème de l'échelle de temps de référence vis-à-vis de l'évolution de la qualité. Les échelles basées sur les stades phénologiques de la mise à fleur ne semblent pas les plus pertinentes, spécialement en situation de sécheresse, où l'on constate au contraire, chez les luzernes pérennes en repousses estivales, une accélération de la floraison et une moindre diminution de la digestibilité. Des études analytiques à l'échelle cellulaire devraient permettre de préciser le degré de liaison existant entre les processus élémentaires de croissance et l'évolution histochimique des parois.

Les teneurs en azote des tiges et des feuilles peuvent être significativement réduites en cas de déficit hydrique. Pour une même composition morphologique, la teneur en azote de la biomasse aérienne peut ainsi être également diminuée par la sécheresse. Cela n'a pas d'influence notable sur la digestibilité, car celle-ci dépend peu de la teneur en azote chez la luzerne.

\section{RÉFÉRENCES}

Albrecht K.A., Wedin W.F. \& Buxton D.R. (1987) Cellwall composition and digestibility of alfalfa stems and leaves. Crop Sci. 27, 735-741

Brown P.W. \& Tanner C.B. (1983) Alfalfa leaf and stem growth during water stress. Agron. J. 75, 799-805

Carter P.R. \& Sheaffer C.C. (1983a) Alfalfa response to soil water deficits. I. Growth, forage quality, yield, water use, and water use efficiency. Crop Sci. 23, 669675
Carter P.R. \&Sheaffer C.C. (1983b) Alfalfa response to soil water deficits. III. Nodulation and $\mathrm{N}_{2}$ fixation. Crop Sci. 23, 985-990

Christian K.R., Jones D.B. \& Freer M. (1970) Digestibility and chemical composition of fractions of lucerne during spring and summer. J. Agric. Sci. Camb. 75, 213-222

Cole D.F., Dobrenz A.K., Massengale M.A. \& Wright L.N. (1970) Water requirements and its association with growth components and protein content of alfalfa (Medicago sativa L.). Crop. Sci. 10, 237-240

Demarquilly C. (1966) Valeur alimentaire de la luzerne. Fourrages 2, 2-33

Durand J-L., Sheehy J.E., Minchin F.R. (1987) Nitrogenase activity, photosynthesis and nodule water poten tial in soyabean plants experiencing water deprivation. J. Exp. Bot. 38, 311-321

Durand J.-L., Lemaire G., Gosse G. \& Chartier M. (1989) Analyse de la conversion de l'énergie solaire en matière sèche par un peuplement de luzerne (Medicago sativa L.) soumis à un déficit hydrique. Agronomie 9, 599-607

Lemaire G. \& Roberge G. (1980) Mise au point d'un dispositif expérimental permettant de suivre la consommation hydrique d'une prairie au cours de sa croissance. Ann. Agron. 31 (4) 455-471

Lemaire G. \& Salette J. (1984) Relation entre dynamique de croissance et dynamique de prélèvement d'azote pour un peuplement de graminées fourragères. I. Etude de l'effet du milieu. Agronomie 4, 423 430

Lemaire G. \& Denoix A. (1987) Croissance estivale en matière sèche de peuplements de fétuque élevée (Festuca arundinacea Schreb.) et de dactyle (Dactylis glomerata L.) dans l'ouest de la France. Il. Interaction entre les niveaux d'alimentation hydrique et de nutrition azotée. Agronomie 7, 381-389

Lemaire G., Cruz P., Gosse G. \& Chartier M. (1985) Etude des relations entre la dynamique de prélèvement d'azote et la dynamique de croissance en matière sèche d'un peuplement de luzerne (Medicago sativa L.). Agronomie 5, 685-692

Salette J. \& Lemaire F. (1981) Sur la variation de la teneur en azote des graminées fourragères pendant leur croissance : formulation d'une loi de dilution. C.R. Acad. Sci Paris, 292, Sér. III, 875-878

Snaydon R.W. (1972) The effect of total water supply, and of frequency of irrigation, upon lucerne. II. Chemical composition. Aust. J. Agric. Res. 23, 253-256

Sprent J.I. (1976) Nitrogen fixation by legumes subject to water and light stresses. In: Symbiotic Nitrogen Fixation by Plants. (P.S. Nutman ed.) Cambridge University Press : Cambridge, pp. 405-420

Tilley J.M.A. \& Terry R.A. (1963) A two-stage technique for the in vitro digestion of forage crops. J. Br. Grassl. Soc. 18, 104-111

Van Soest P.J. (1963) Use of detergents in the analysis of fibrous feeds. II. A rapid method for the determination of fiber and lignin. J. Assoc. Offic. Anal. Chem., 46, 829-835

Vough L.R. \& Marten G.C. (1971) The influence of soil moisture and ambient temperature on yield and quality of alfalfa forage. Agron. J. 63 (1) 40-42

Wery J. (1987) Relations entre la nutrition azotée et la production chez les légumineuses. In : Nutrition Azotée des Légumineuses, INRA, Paris, pp. 199-223 